\title{
SPECTRALLY ENCODED COMMON-PATH FIBER-OPTIC-BASED PARALLEL OPTICAL COHERENCE TOMOGRAPHY
}

\author{
Kye-Sung Lee ${ }^{+*}$, Hwan Hur, I Jong Kim, Geon-Hee Kim \\ Korea Basic Science Institute, Yuseong-gu, Daejeon, South Korea

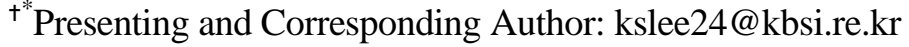

\begin{abstract}
We recently reported a fiber-optic-based parallel optical coherence tomography (OCT) using spectrally encoded extended illumination with a common-path handheld probe, where the flexibility and robustness of the system are significantly improved, which is critical in the clinical environment. In there, we also investigated the effect of the phase stability of the fiber-based interferometry on the parallel OCT system by comparing the common-path OCT with two-arm OCT. We also showed the phase stability was 32 times better than that of the two-arm OCT using a homemade common-path handheld probe based on a Mirau interferometer. In result, the axial resolution of the common-path OCT was measured as $5.1 \pm 0.3 \mu \mathrm{m}$ and human skin was imaged to demonstrate the in vivo imaging performance of the fiber-optic-based parallel OCT. In presentation, furthermore, we will demonstrate progress up to now in addition to the result.
\end{abstract}

KEYWORDS: Optical coherence tomography, Fiber optics imaging, Dermatology, Medical optics instrumentation 